PROCEEDINGS OF THE

AMERICAN MATHEMATICAL SOCIETY

Volume 127, Number 7, Pages 2155-2162

S 0002-9939(99)05220-X

Article electronically published on March 3, 1999

\title{
HELLY-TYPE THEOREMS FOR HOLLOW AXIS-ALIGNED BOXES
}

\author{
KONRAD J. SWANEPOEL
}

(Communicated by Jeffry N. Kahn)

\begin{abstract}
A hollow axis-aligned box is the boundary of the cartesian product of $d$ compact intervals in $\mathbb{R}^{d}$. We show that for $d \geq 3$, if any $2^{d}$ of a collection of hollow axis-aligned boxes have non-empty intersection, then the whole collection has non-empty intersection; and if any 5 of a collection of hollow axis-aligned rectangles in $\mathbb{R}^{2}$ have non-empty intersection, then the whole collection has non-empty intersection. The values $2^{d}$ for $d \geq 3$ and 5 for $d=2$ are the best possible in general. We also characterize the collections of hollow boxes which would be counterexamples if $2^{d}$ were lowered to $2^{d}-1$, and 5 to 4 , respectively.
\end{abstract}

\section{General notation and Definitions}

We denote the cardinality of a set $S$ by $\# S$. Let $\Pi(\mathbf{S}, k)$ denote the property that any subcollection of $\mathbf{S}$ of at most $k$ sets has non-empty intersection (where $k$ is any positive integer), and $\Pi(\mathbf{S})$ the property that $\mathbf{S}$ has non-empty intersection. For any set $S \subseteq \mathbb{R}^{d}$, we denote the convex hull, interior and boundary by co $S$, int $S$ and bd $S$, respectively. An axis-aligned box in $\mathbb{R}^{d}$ is the cartesian product of $d$ compact intervals, i.e. a set of the form

$$
\prod_{i=1}^{d}\left[a_{i}, b_{i}\right]=\left\{\left(x_{1}, \ldots, x_{d}\right) \in \mathbb{R}^{d}: a_{i} \leq x_{i} \leq b_{i}, i=1, \ldots, d\right\} \quad\left(a_{i}<b_{i}\right) .
$$

An axis-aligned hollow box in $\mathbb{R}^{d}$ is the boundary of a box, i.e. a set of the form

$$
\operatorname{bd} \prod_{i=1}^{d}\left[a_{i}, b_{i}\right] \quad\left(a_{i}<b_{i}\right) .
$$

In the rest of the paper, the word axis-aligned is implicit whenever we refer to boxes or hollow boxes. In the next section we state our results (Theorems 1 and 2), together with examples showing that they are the best possible. In Section 3 we derive a combinatorial lemma needed in the proofs of these theorems in Section 4.

Received by the editors October 15, 1997.

1991 Mathematics Subject Classification. Primary 52A35.

Key words and phrases. Helly-type theorem, box, cube, hypercube.

(C)1999 American Mathematical Society 


\section{Helly-type theOREMS}

A Helly-type theorem may be loosely described as an analogue of

Helly's Theorem ([6]). Let $\mathbf{S}$ be a collection of convex sets in $\mathbb{R}^{d}$ that is finite or contains at least one compact set. Then

$$
\Pi(\mathbf{S}, d+1) \Longrightarrow \Pi(\mathbf{S}) .
$$

There is an abundance of literature on Helly-type theorems; see the surveys $[1,3,5]$. Most of these analogues consider collections of convex sets, exactly as in Helly's Theorem. Here are two examples where non-convex sets are considered.

Theorem (Motzkin $[8,2]$ ). Let $\mathbf{S}$ be a collection of sets in $\mathbb{R}^{d}$, each of which is the set of common zeroes of a set of real polynomials in $d$ variables of degree at most $k$. Then

$$
\Pi\left(\mathbf{S},\left(\begin{array}{c}
d+k \\
k
\end{array}\right)\right) \Longrightarrow \Pi(\mathbf{S}) .
$$

Theorem (Maehara $[7,4]$ ). Let $\mathbf{S}$ be a collection of at least $d+3$ euclidean spheres in $\mathbb{R}^{d}$. Then

$$
\Pi(\mathbf{S}, d+1) \Longrightarrow \Pi(\mathbf{S}) .
$$

In both of these theorems the sets are algebraic. In this paper we find Hellytype theorems for certain non-algebraic sets, namely hollow boxes. It is well known (and immediately follows from the one-dimensional Helly Theorem) that for any collection $\mathbf{S}$ of boxes in $\mathbb{R}^{d}$,

$$
\Pi(\mathbf{S}, 2) \Longrightarrow \Pi(\mathbf{S}) \text {. }
$$

If we want the boxes to intersect only in their boundaries, then the value 2 has to be greatly enlarged, as the following examples show.

Example 1. A class of collections $\mathbf{S}$ of hollow boxes in $\mathbb{R}^{d}$ such that $\Pi(\mathbf{S}, 2 d)$ holds, but $\operatorname{not} \Pi(\mathbf{S}, 2 d+1)$.

Choose any box $B=\prod_{i=1}^{d}\left[x_{i}^{0}, x_{i}^{1}\right]$ (where $\left.x_{i}^{0}<x_{i}^{1}\right)$, and $p=\left(p_{1}, \ldots, p_{d}\right) \in$ int $B$. For $i=1, \ldots, d$ and $j=0,1$, let $F_{i}^{j}$ denote the facet of $B$ contained in the hyperplane $\left\{x \in \mathbb{R}^{d}: x_{i}=x_{i}^{j}\right\}$. Let $\mathbf{S}$ be any collection of hollow boxes such that

(1) $\operatorname{bd} B \in \mathbf{S}$,

(2) $p \in D$ for all $D \in \mathbf{S} \backslash\{\mathrm{bd} B\}$,

(3) for each $D \in \mathbf{S} \backslash\{\operatorname{bd} B\}$ there is a facet of $B$ contained in $D$,

(4) for each facet $F$ of $B$ there exists some $D \in \mathbf{S} \backslash\{\mathrm{bd} B\}$ such that $F \subseteq D$.

It is clear that there exist such collections $\mathbf{S}$ (even infinite ones, provided that $d \neq 1)$. Note that the facet in (3) is unique, by (2). See Figure 1 for an example in $\mathbb{R}^{2}$.

Choose any subcollection $\mathbf{T} \subseteq \mathbf{S}$ of $2 d$ hollow boxes. If bd $B \notin \mathbf{T}$, then by (2), $\bigcap_{D \in \mathbf{T}} D \neq \emptyset$. Otherwise, by (3), there is a facet of $B$ not contained in any $D \in$ $\mathbf{T} \backslash\{$ bd $B\}$, say $F_{1}^{0}$. Then it easily follows from (2) and (3) that $\left(x_{1}^{1}, p_{2}, p_{3}, \ldots, p_{d}\right) \in$ $\bigcap_{D \in \mathbf{T}} D$. It follows that $\Pi(\mathbf{S}, 2 d)$ holds.

Secondly, use (4) to choose for each facet $F_{i}^{j}$ of $B$ a $D_{i}^{j} \in \mathbf{S}$ containing $F_{i}^{j}$. Then $F_{i}^{1-j} \cap D_{i}^{j}=\emptyset$ by $(2)$. It follows that $(\operatorname{bd} B) \cap \bigcap_{i=1}^{d}\left(D_{i}^{0} \cap D_{i}^{1}\right)=\emptyset$, and $\Pi(\mathbf{S}, 2 d+1)$ does not hold. 


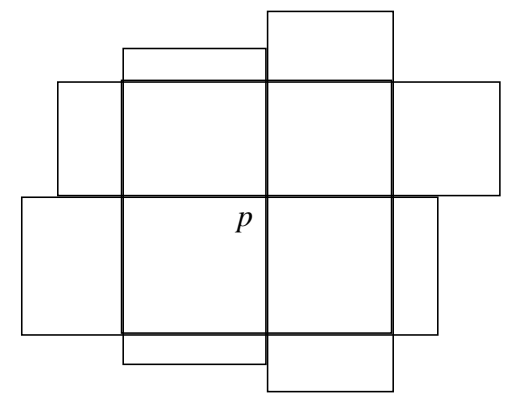

FiguRe 1. Five rectangles with no common boundary point, yet any 4 have a common boundary point

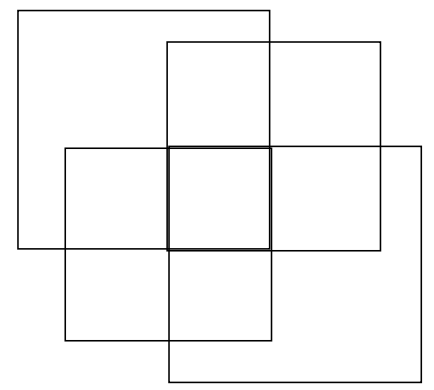

FigURE 2. Four rectangles with no common boundary point, yet any 3 have a common boundary point

Example 2. A class of collections $\mathbf{S}$ of hollow boxes in $\mathbb{R}^{d}$ such that $\Pi\left(\mathbf{S}, 2^{d}-1\right)$ holds, but not $\Pi\left(\mathbf{S}, 2^{d}\right)$.

Let $B=\prod_{i=1}^{d}\left[x_{i}^{0}, x_{i}^{1}\right]\left(x_{i}^{0}<x_{i}^{1}\right)$, and let $\mathbf{S}$ be any collection of hollow boxes such that

$$
\begin{aligned}
& B \subseteq \operatorname{co} D \text { for all } D \in \mathbf{S}, \\
& \text { for each vertex } v \text { of } B \text { there exists a } D \in \mathbf{S} \text { not containing } v, \\
& \text { each } D \in \mathbf{S} \text { contains all the vertices of } B \text { except at most one. }
\end{aligned}
$$

Thus it is clear there exist such collections, even infinite ones. See Figure 2 for an example in $\mathbb{R}^{2}$. Given a subcollection of $2^{d}-1$ hollow boxes, then by (7) some vertex of $B$ is contained in all these boxes. Thus $\Pi\left(\mathbf{S}, 2^{d}-1\right)$ holds.

Secondly, (6) gives a subcollection of $2^{d}$ boxes $D_{v}$ with $v \notin D_{v}$. But then, also using (5), it follows from

Lemma 4.2 that for any vertex $w$ of $B, \bigcap_{v \neq w} D_{v}=\{w\}$. Thus, $\bigcap_{v} D_{v}=\emptyset$, and $\Pi\left(\mathbf{S}, 2^{d}\right)$ does not hold.

The following two theorems show that the collections in Example 1 in the case $d=2$, and the collections in Example 2 in the case $d \geq 3$ are the worst cases.

Theorem 1. Let $\mathbf{S}$ be a collection of hollow boxes in $\mathbb{R}^{2}$. Then

$$
\Pi(\mathbf{S}, 5) \Longrightarrow \Pi(\mathbf{S}) \text {. }
$$


If $\mathbf{S}$ is furthermore not of the form in Example 1, then

$$
\Pi(\mathbf{S}, 4) \Longrightarrow \Pi(\mathbf{S}) \text {. }
$$

Theorem 2. Let $d \geq 3$, and $\mathbf{S}$ a collection of hollow boxes in $\mathbb{R}^{d}$. Then

$$
\Pi\left(\mathbf{S}, 2^{d}\right) \Longrightarrow \Pi(\mathbf{S}) \text {. }
$$

If $\mathbf{S}$ is furthermore not of the form in Example 2, then

$$
\Pi\left(\mathbf{S}, 2^{d}-1\right) \Longrightarrow \Pi(\mathbf{S}) \text {. }
$$

Note that in $\mathbb{R}^{1}$, a hollow box is a two-point set. It is trivially seen that for a collection $\mathbf{S}$ of two-point sets,

$$
\Pi(\mathbf{S}, 2) \Longrightarrow \Pi(\mathbf{S})
$$

except if $\mathbf{S}=\{\{a, b\},\{b, c\},\{c, a\}\}$ for some distinct elements $a, b, c$, i.e. if $\mathbf{S}$ is as in Example 1.

\section{Combinatorial preparation}

A string of length $d$ over the alphabet $A$ is any $d$-tuple from $A^{d}$, and is written as $\varepsilon=\varepsilon_{1} \ldots \varepsilon_{d}$. We say that $\varepsilon_{i}$ is in position $i$. A pattern is a string over $\{0,1, *\}$. A string $\varepsilon_{1} \ldots \varepsilon_{d}$ over $\{0,1\}$ matches a pattern $\rho_{1} \ldots \rho_{d}$ if for all $i=1, \ldots, d$, $\rho_{i}=0 \Rightarrow \varepsilon_{i}=0$ and $\rho_{i}=1 \Rightarrow \varepsilon_{i}=1$. Thus, a $*$ in a pattern is a "wildcard" matching 0 or 1 . A cover of $\{0,1\}^{d}$ is a set of patterns $\mathbf{C} \subseteq\{0,1, *\}^{d}$ such that any string in $\{0,1\}^{d}$ matches some pattern in C. A minimal cover of $\{0,1\}^{d}$ is a cover $\mathbf{C}$ of $\{0,1\}^{d}$ such that no proper subset of $\mathbf{C}$ is a cover of $\{0,1\}^{d}$.

Lemma 1. Let $\mathbf{C}$ be a minimal cover of $\{0,1\}^{d}$. Then, for each $i=1, \ldots, d$, $E_{i}:=\left\{\varepsilon_{i}: \varepsilon_{1} \ldots \varepsilon_{d} \in C\right\}$ is equal to either $\{*\},\{0,1\}$ or $\{0,1, *\}$. Let $s:=$ $\#\left\{i: E_{i}=\{*\}\right\}$. Then $\# \mathbf{C} \leq 2^{d-s}$, with equality iff $\mathbf{C}=\left\{\varepsilon: \varepsilon_{i}=*\right.$ for all $\left.i \in J\right\}$ for some $J \subseteq\{1,2, \ldots, d\}$ with $\# J=s$.

Proof. We first show that any minimal cover $\mathbf{C}$ satisfies $\# \mathbf{C} \leq 2^{d}$, with equality iff $\mathbf{C}=\{0,1\}^{d}$. For each pattern $\rho \in \mathbf{C}$, the set $\mathbf{C} \backslash\{\rho\}$ is not a cover of $\{0,1\}^{d}$, and there exists a string $\varepsilon_{\rho} \in\{0,1\}^{d}$ that matches $\rho$ but does not match any other pattern in C. Thus,

$$
\phi: \mathbf{C} \rightarrow\{0,1\}^{d} ; \rho \mapsto \varepsilon_{\rho}
$$

is an injection, and $\# \mathbf{C} \leq 2^{d}$. If equality holds, $\phi$ is a bijection, and any string in $\{0,1\}^{d}$ matches a unique pattern in $\mathbf{C}$. Thus $\mathbf{C}$ defines a partition of $\{0,1\}^{d}$ : a block of the partition consists of all strings matching a given pattern in $\mathbf{C}$. Since there are $2^{d}$ blocks, each block must contain exactly 1 element. Thus no pattern in $\mathbf{C}$ contains a $*$, and $\mathbf{C}=\{0,1\}^{d}$.

Secondly, we show that if 0 does not occur in the first position of any string in $\mathbf{C}$, there are only $*$ 's in the first position. Let

$$
\mathbf{C}^{*}=\left\{\varepsilon_{2} \ldots \varepsilon_{n}: * \varepsilon_{2} \ldots \varepsilon_{n} \in \mathbf{C}\right\} .
$$

It is easily seen that $\mathbf{C}^{*}$ is a cover for $\{0,1\}^{d-1}$ : For any $\varepsilon \in\{0,1\}^{d-1}, 0 \varepsilon$ matches some pattern in $\mathbf{C}$ starting with $*$. But then, by putting back $*$ in the first position of every pattern in $\mathbf{C}^{*}$, we already obtain a cover of $\{0,1\}^{d}$. Thus, 1 does not occur in the first position in any string in C. Similarly, if 1 does not occur in the first position, then there are again only $*$ 's in the first position. 
Finally, to complete the proof, delete the positions for which $E_{i}=\{*\}$, to obtain $\mathbf{C}^{\prime} \subseteq\{0,1, *\}^{d-s}$. Then $\mathbf{C}^{\prime}$ is clearly a minimal cover of $\{0,1\}^{d-s}$, and $\# \mathbf{C}=\# \mathbf{C}^{\prime}$. Now apply the first part of the proof.

We omit the proof of the following elementary inequality.

Lemma 2. Let $d \geq s \geq 0$ be integers. Then $2^{d-s}<2^{d}-2 s$, except in the following cases:

1. If $(d, s)=(1,1)$ or $(d, s)=(2,2)$, the opposite inequality holds.

2. If $s=0$, or $(d, s)=(2,1)$, there is equality.

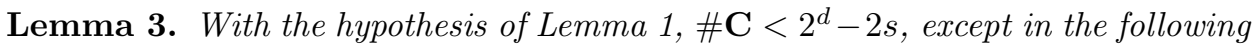
cases:

1. If $\mathbf{C}=\{*\}$ or $\mathbf{C}=\{* *\}$, then $\# \mathbf{C}>2^{d}-2 s=0$.

2. If $\mathbf{C}=\{0,1\}^{d}$ or $\mathbf{C}=\{0 *, 1 *\}$ or $\mathbf{C}=\{* 0, * 1\}$, then $\# \mathbf{C}=2^{d}-2 s$.

Proof. It is easy to check everything for $d=1$ and $d=2$ : The only minimal covers for $d=1$ are $\{*\}$ and $\{0,1\}$, and for $d=2$, are equivalent (up to permutation of the positions, and interchange of 0 and 1 ) to one of

$$
\{* *\},\{0 *, 1 *\},\{0 *, 10,11\},\{0 *, * 0,11\},\{00,01,10,11\} .
$$

For $d \geq 3$, if $s \geq 1$, then $\# \mathbf{C} \leq 2^{d-s}<2^{d}-2 s$, by Lemmas 1 and 2. Otherwise, $s=0$, and by Lemma $1, \# \mathbf{C}<2^{d}$ unless $\mathbf{C}=\{0,1\}^{d}$.

\section{Proofs of Theorems 1 And 2}

We first prove a rather technical lemma, which gives some insight into the (not easily visualizable) intersections of hollow boxes.

Lemma 4. Let $B=\prod_{i=1}^{d}\left[x_{i}^{0}, x_{i}^{1}\right]$, with $x_{i}^{0} \leq x_{i}^{1}$ for each $i=1, \ldots, d$. (Thus $B$ is not necessarily full-dimensional.) For each string $\varepsilon \in\{0,1\}^{d}$, let $x_{\varepsilon}:=$ $\left(x_{1}^{\varepsilon_{1}}, x_{2}^{\varepsilon_{2}}, \ldots, x_{d}^{\varepsilon_{d}}\right)$, and let $D_{\varepsilon}$ be a hollow box such that $x_{\varepsilon} \notin D_{\varepsilon}$ and $B \subseteq \operatorname{co} D_{\varepsilon}$. (Thus $\left\{x_{\varepsilon}: \varepsilon \in\{0,1\}^{d}\right\}$ is the vertex set of $B$, with repetitions if $\operatorname{dim} B<d$.) Then,

1. $B \cap \bigcap_{\varepsilon} D_{\varepsilon}=\emptyset$,

2. for any $\gamma \in\{0,1\}^{d}, B \cap \bigcap_{\varepsilon \neq \gamma} D_{\varepsilon} \subseteq\left\{x_{\gamma}\right\}$,

3. for any $\gamma, \delta \in\{0,1\}^{d}$,

$$
B \cap \bigcap_{\varepsilon \neq \gamma, \delta} D_{\varepsilon} \subseteq \begin{cases}\operatorname{co}\left\{x_{\gamma}, x_{\delta}\right\} & \text { if } x_{\gamma} \text { and } x_{\delta} \text { differ in exactly one coordinate, } \\ \left\{x_{\gamma}, x_{\delta}\right\} & \text { otherwise. }\end{cases}
$$

Proof. Clearly, part 1 follows from part 2: If $B$ is a single point, each $D_{\varepsilon}$ is disjoint from $B$. Otherwise, choose $\gamma, \gamma^{\prime}$ such that $x_{\gamma} \neq x_{\gamma^{\prime}}$. Then, by part $2, B \cap \bigcap_{\varepsilon} D_{\varepsilon}=\emptyset$.

Although part 2 also easily follows from part 3 , we first prove part 2 , as it clears the way for a proof of part 3 . For each $\varepsilon$, write $D_{\varepsilon}=\operatorname{bd} \prod_{i=1}^{d}\left[a_{i}^{\varepsilon}, b_{i}^{\varepsilon}\right]$. Let $x=\left(x_{1}, x_{2}, \ldots, x_{d}\right) \in B \cap \bigcap_{\varepsilon \neq \gamma} D_{\varepsilon}$. Then $x_{i}^{0} \leq x_{i} \leq x_{i}^{1}$ for each $i$. Define $\varepsilon$ by

$$
\varepsilon_{i}:= \begin{cases}\gamma_{i} & \text { if } x_{i}=x_{i}^{\gamma_{i}} \\ 1-\gamma_{i} & \text { otherwise. }\end{cases}
$$

Since $x_{\varepsilon} \subseteq B \subseteq \operatorname{co} D_{\varepsilon}$, but $x_{\varepsilon} \notin D_{\varepsilon}$, we have $a_{i}^{\varepsilon} \leq x_{i}^{0} \leq x_{i}^{1} \leq b_{i}^{\varepsilon}$ and $a_{i}^{\varepsilon}<x_{i}^{\varepsilon_{i}}<b_{i}^{\varepsilon}$ for all $i$. If $\varepsilon_{i}=\gamma_{i}$, then $x_{i}^{\varepsilon_{i}}=x_{i}^{\gamma_{i}}=x_{i}$. If $\varepsilon_{i}=1-\gamma_{i}$, then $x_{i} \neq x_{i}^{\gamma_{i}}$, and either 
$\gamma_{i}=1$ and $x_{i}^{\varepsilon_{i}}=x_{i}^{0} \leq x_{i}<x_{i}^{1}$, or $\gamma_{i}=0$ and $x_{i}^{\varepsilon_{i}}=x_{i}^{1} \geq x_{i}>x_{i}^{0}$. In all cases, $a_{i}^{\varepsilon}<x_{i}<b_{i}^{\varepsilon}$, and it follows that $x \notin D_{\varepsilon}$. Thus $\varepsilon=\gamma$, and $x_{i}=x_{i}^{\gamma_{i}}$ for all $i$. It follows that $x=x_{\gamma}$.

Now let $x \in B \cap \bigcap_{\varepsilon \neq \gamma, \delta} D_{\varepsilon}$, and suppose $x \neq x_{\gamma}, x_{\delta}$. Let $j$ be any position such that $x_{j} \neq x_{j}^{\gamma_{j}}$. Define $\varepsilon$ by

$$
\varepsilon_{i}:= \begin{cases}1-\gamma_{i} & \text { if } i=j, \\ \delta_{i} & \text { if } x_{i}=x_{i}^{\delta_{i}}, i \neq j, \\ 1-\delta_{i} & \text { if } x_{i} \neq x_{i}^{\delta_{i}}, i \neq j .\end{cases}
$$

As in the proof of part 2, for each $i$ we obtain $a_{i}^{\varepsilon}<x_{i}<b_{i}^{\varepsilon}$, and therefore, $x \notin D_{\varepsilon}$. Thus, $\varepsilon=\gamma$ or $\varepsilon=\delta$. But, since $\varepsilon_{j} \neq \gamma_{j}$, we must have $\varepsilon=\delta$. Thus, $\gamma_{j}=1-\delta_{j}$, and for all $i \neq j, x_{i}=x_{i}^{\delta_{i}}$. Since $x \neq x_{\delta}$, we then must have $x_{j} \neq x_{j}^{\delta_{j}}$. By repeating the above argument with $x_{\delta}$ instead of $x_{\gamma}$, we also obtain that for all $i \neq j, x_{i}=x_{i}^{\gamma_{i}}$. It follows that $x \in \operatorname{co}\left\{x_{\gamma}, x_{\delta}\right\}$, and $x_{\gamma}$ and $x_{\delta}$ differ in only one coordinate.

Proof of Theorem 2. Note that the first part of the theorem follows from the second part, since $\Pi\left(\mathbf{S}, 2^{d}\right)$ does not hold in Example 2. By compactness, we only have to prove the theorem for finite $\mathbf{S}$. We assume that $\Pi\left(\mathbf{S}, 2^{d}-1\right)$. Let $B=\bigcap_{D \in \mathbf{S}} \operatorname{co} D=$ $\prod_{i=1}^{d}\left[x_{i}^{0}, x_{i}^{1}\right]$. (Since any two $D$ 's intersect, $x_{i}^{0} \leq x_{i}^{1}$ for all $i$.) We denote the vertices of $B$ by $x_{\varepsilon}, \varepsilon \in\{0,1\}^{d}$, as in Lemma 4 . We now show that if $x_{\varepsilon} \notin \bigcap_{D \in \mathbf{S}} D$ for all $\varepsilon$, then $\mathbf{S}$ is as in Example 2.

For each $\varepsilon$, choose $D_{\varepsilon}=\operatorname{bd} \prod_{i=1}^{d}\left[a_{i}^{\varepsilon}, b_{i}^{\varepsilon}\right] \in \mathbf{S}$ such that $x_{\varepsilon} \notin D_{\varepsilon}$, and let

$$
X_{\varepsilon}:=\left\{x_{\delta}: \delta \in\{0,1\}^{d}, x_{\delta} \notin D_{\varepsilon}\right\} .
$$

Then $X_{\varepsilon}=\left\{x_{\delta}: \delta\right.$ matches $\left.\rho_{\varepsilon}\right\}$, where $\rho_{\varepsilon}=\rho_{1} \ldots \rho_{d}$ is the pattern defined by

$$
\rho_{i}:= \begin{cases}0 & \text { if } a_{i}^{\varepsilon}<x_{i}^{0} \text { and } x_{i}^{1}=b_{i}^{\varepsilon}, \\ 1 & \text { if } a_{i}^{\varepsilon}=x_{i}^{0} \text { and } x_{i}^{1}<b_{i}^{\varepsilon}, \\ * & \text { if } a_{i}^{\varepsilon}<x_{i}^{0} \text { and } x_{i}^{1}<b_{i}^{\varepsilon} .\end{cases}
$$

Thus $\mathbf{C}:=\left\{\rho_{\varepsilon}: \varepsilon \in\{0,1\}^{d}\right\}$ is a cover of $\{0,1\}^{d}$. If $\rho_{\varepsilon}=\rho_{\varepsilon^{\prime}}$, then $x_{\varepsilon^{\prime}} \notin D_{\varepsilon}$, so we may choose the $D_{\varepsilon}$ 's such that if $\rho_{\varepsilon}=\rho_{\varepsilon^{\prime}}$, then $D_{\varepsilon}=D_{\varepsilon^{\prime}}$. We now write $D_{\rho}$ for $D_{\varepsilon}$ whenever $\rho=\rho_{\varepsilon} \in \mathbf{C}$. Let $\mathbf{C}^{\prime}$ be a minimal cover contained in $\mathbf{C}$. For each $\varepsilon \in\{0,1\}^{d}$ there now exists a $\rho \in \mathbf{C}^{\prime}$ matching $\varepsilon$ such that $x_{\varepsilon} \notin D_{\rho}$. Applying Lemma 4.1 to $\left\{D_{\rho}: \rho \in \mathbf{C}^{\prime}\right\}$, we find $B \cap \bigcap_{\rho} D_{\rho}=\emptyset$. Let $J \subseteq\{1, \ldots, d\}$ be the set of positions in which there are only $*$ 's in $\mathbf{C}^{\prime}$. For each $j \in J$, choose $D_{j}^{0}=\operatorname{bd} \prod_{i=1}^{d}\left[r_{i}^{j}, s_{i}^{j}\right]$ and $D_{j}^{1}=\operatorname{bd} \prod_{i=1}^{d}\left[t_{i}^{j}, u_{i}^{j}\right]$ from $\mathbf{S}$ such that $r_{j}^{j}=x_{j}^{0}$ and $u_{j}^{j}=x_{j}^{1}$ (which is possible since $\mathbf{S}$ is finite). Since (by Lemma 1 ) for each $i \notin J$ there exist $\rho, \rho^{\prime} \in \mathbf{C}^{\prime}$ such that $\rho_{i}=0$ and $\rho_{i}^{\prime}=1$, we obtain

$$
\bigcap_{j \in J}\left(\operatorname{co} D_{j}^{0} \cap \operatorname{co} D_{j}^{1}\right) \cap \bigcap_{\rho \in \mathbf{C}^{\prime}} \operatorname{co} D_{\rho}=B .
$$

Thus, letting $\mathbf{T}:=\left\{D_{\rho}: \rho \in \mathbf{C}^{\prime}\right\} \cup\left\{D_{j}^{0}, D_{j}^{1}: j \in J\right\}$, we obtain $\bigcap_{D \in \mathbf{T}} D=\emptyset$. Thus, $\# \mathbf{T} \geq 2^{d}$. Also, $\# \mathbf{T} \leq \# \mathbf{C}^{\prime}+2 \# J$. Thus, by Lemma $3, \mathbf{C}^{\prime}=\{0,1\}^{d}$. It follows that $x_{\delta} \notin D_{\varepsilon}$ iff $\delta=\varepsilon$. Thus, all $x_{\varepsilon}$ 's are distinct, and $B$ is full-dimensional. Also, $J=\emptyset$ and $B=\bigcap_{\varepsilon} \operatorname{co} D_{\varepsilon}$. In fact, if we take any $\varepsilon$ and $\varepsilon^{\prime}$ which differ in each position, then $B=\operatorname{co} D_{\varepsilon} \cap \operatorname{co} D_{\varepsilon^{\prime}}$. 


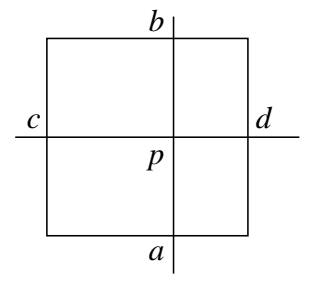

FiguRE 3

We already have that $\mathbf{S}$ satisfies (5) and (6) in Example 2. Consider any $D \in \mathbf{S}$ with $D \neq D_{\varepsilon}$ for all $\varepsilon$. Suppose there exist distinct $\gamma, \delta$ such that $x_{\gamma}, x_{\delta} \notin D$. By Lemma 4.3, $D \cap B \cap \bigcap_{\varepsilon \neq \gamma, \delta} D_{\varepsilon}=\emptyset$. But there exist $\varepsilon, \varepsilon^{\prime} \notin\{\gamma, \delta\}$ differing in each position. Thus $\bigcap_{\varepsilon \neq \gamma, \delta} D_{\varepsilon} \subseteq B$, and $D \cap \bigcap_{\varepsilon \neq \gamma, \delta} D_{\varepsilon}=\emptyset$, contradicting $\Pi\left(\mathbf{S}, 2^{d}-1\right)$. Thus $D$ contains all $x_{\varepsilon}$ 's, except at most one and (7) is satisfied.

Proof of Theorem 1. Proceeding as in the proof of Theorem 2, we assume that $\Pi(\mathbf{S}, 4)$ holds and that no vertex of $B$ is in $\bigcap_{D \in \mathbf{S}} D$, and obtain $\mathbf{C}^{\prime}=\{* *\}$ and $\# \mathbf{T}=5$.

We now show that $\mathbf{S}$ is as in Example 1. Since $\mathbf{C}^{\prime}=\{* *\}$, there is only one $D_{\rho}$, say $D=D_{* *}$, which is disjoint from $B$. Also, $\mathbf{T}=\left\{D_{1}^{0}, D_{1}^{1}, D_{2}^{0}, D_{2}^{1}, D\right\}$, with the $D_{j}^{i}$ s s as in the proof of Theorem 2. Thus $\bigcap_{i, j} \operatorname{co} D_{j}^{i}=B$.

Suppose that for each $\varepsilon \in\{0,1\}^{2}$ there exists a $D_{j}^{i}$ not containing $x_{\varepsilon}$. Then by Lemma $4.1, \bigcap_{i, j} D_{j}^{i}=\emptyset$, contradicting $\Pi(\mathbf{S}, 4)$. Thus, some $x_{\varepsilon} \in \bigcap_{i, j} D_{j}^{i}$, say $x_{00}$.

Suppose that $B$ is two-dimensional, i.e. $x_{1}^{0}<x_{1}^{1}$ and $x_{2}^{0}<x_{2}^{1}$. Then, since $x_{00} \in$ $D_{1}^{1}, D_{1}^{1}$ contains at least two sides of $B$, and it follows that $B=\operatorname{co} D_{1}^{1} \cap \operatorname{co} D_{2}^{0} \cap \operatorname{co} D_{2}^{1}$ or $B=\operatorname{co} D_{1}^{1} \cap \operatorname{co} D_{1}^{0} \cap \operatorname{co} D_{2}^{1}$. Thus

$$
D_{1}^{1} \cap D_{2}^{0} \cap D_{2}^{1} \cap D=\emptyset \quad \text { or } \quad D_{1}^{1} \cap D_{1}^{0} \cap D_{2}^{1} \cap D=\emptyset,
$$

both cases contradicting $\Pi(\mathbf{S}, 4)$.

Suppose $B$ is one-dimensional, say $x_{1}^{0}<x_{1}^{1}$ and $x_{2}^{0}=x_{2}^{1}$. Then $D_{2}^{0} \cap D_{2}^{1}$ is a horizontal segment containing $B$. If $D_{1}^{1}$ intersects $D_{2}^{0} \cap D_{2}^{1}$ only in $x_{00}$ and $x_{10}$, then $D_{1}^{1} \cap D_{2}^{0} \cap D_{2}^{1} \cap D=\emptyset$, a contradiction. Thus, $B \subseteq D_{1}^{1}$. We may assume that $D_{2}^{1}$ and $D_{1}^{1}$ are on opposite sides of $B$ (otherwise consider $D_{2}^{0}$ and $D_{1}^{1}$ ). Then

$$
D_{1}^{0} \cap D_{1}^{1} \cap D_{2}^{1} \subseteq B \quad \text { and } \quad D_{1}^{0} \cap D_{1}^{1} \cap D_{2}^{1} \cap D=\emptyset,
$$

a contradiction.

Thus $B$ is zero-dimensional, say $B=\{p\}$, where $p=x_{00}=\left(x_{1}, x_{2}\right)$ and $x_{1}=$ $x_{1}^{0}=x_{1}^{1}, x_{2}=x_{2}^{0}=x_{2}^{1}$. Then $D_{1}^{0} \cap D_{1}^{1}$ is a vertical line segment through $p$ which must intersect $D_{2}^{0} \cap D$ in a point $b \neq p$, and $D_{2}^{1} \cap D$ in a point $a \neq p$. Similarly, $D_{1}^{0} \cap D_{1}^{1}$ is a horizontal segment through $p$ which must intersect $D_{1}^{0} \cap D$ in a point $d \neq p$, and $D_{1}^{1} \cap D$ in a point $c \neq p$. See Figure 3. Now $\mathbf{S}$ already satisfies (1) and (4) of Example 1, if we take $B$ there as co $D$.

Consider any $E \in \mathbf{S} \backslash \mathbf{T}$. By considering the intersection of three sets at a time from $\mathbf{T}$, we see that $E$ must intersect each of the sets $\{a, b\},\{c, d\},\{p, a\},\{p, b\}$, $\{p, c\},\{p, d\}$. If $p \notin E$, then $a, b, c, d \in E$, and $E=D$, a contradiction.

Thus $p \in E$, and (2) is satisfied. Also, $a \in E$ or $b \in E$. We may assume without loss that $a \in E$, and similarly, $c \in E$. But then, since $E \cap D \cap D_{2}^{0} \cap D_{1}^{0} \neq \emptyset$, we 
must have either $b \in E$ or $d \in E$, and (3) is satisfied. It follows that $\mathbf{S}$ is as in Example 1.

\section{ACKNOWLEDGEMENT}

This paper is based on part of the author's Ph.D. thesis written under supervision of Professor W. L. Fouché at the University of Pretoria. I thank the referee for pointing out a few small errors in a previous version of this paper.

\section{REFERENCES}

[1] L. Danzer, B. Grünbaum, and V. Klee, Helly's theorem and its relatives, Convexity (V. L. Klee, ed.), Proc. of Symposia in Pure Math., vol. 7, A.M.S., 1963, pp. 100-181. MR 28:524

[2] M. Deza and P. Frankl, A Helly type theorem for hypersurfaces, J. Comb. Theory, Ser. A 45 (1987), 27-30. MR 88e:52012

[3] J. Eckhoff, Helly, Radon, and Carathéodory type theorems, Handbook of Convex Geometry (P. M. Gruber and J. M. Wills, eds.), Elsevier Science Publishers B.V., 1993, pp. 389-448. MR 94k:52010

[4] P. Frankl, Helly-type theorems for varieties, Europ. J. Combinatorics 10 (1989), 243-245. MR 90k:52014

[5] J. E. Goodman, R. Pollack, and R. Wenger, Geometric transversal theory, New Trends in Discrete and Computational Geometry (J. Pach, ed.), Springer-Verlag, Heidelberg, 1993. MR 95c: 52010

[6] E. Helly, Über Mengen konvexer Körper mit gemeinschaftlichen Punkten, Jber. Deutsch. Math. Verein 32 (1923), 175-176.

[7] H. Maehara, Helly-type theorems for spheres, Discrete Comp. Geom. 4 (1989), 279-285. MR 90c: 52016

[8] T. S. Motzkin, A proof of Hilbert's Nullstellensatz, Math. Zeitschr. 63 (1955), 341-344.

Department of Mathematics and Applied Mathematics, University of Pretoria, PreTORIA 0002, SOUTh AFrica

E-mail address: konrad@math.up.ac.za 